www.jmscr.igmpublication.org

Impact Factor 5.84

Index Copernicus Value: 71.58

ISSN (e)-2347-176x ISSN (p) 2455-0450

crossref DOI: _https://dx.doi.org/10.18535/jmscr/v6i1.43

Journal Of Medical Science And Clinical Research

\title{
Anti Rabies Vaccination compliance: A longitudinal study amongst patients attending Anti Rabies Vaccination OPD in a tertiary care hospital
}

\author{
Authors \\ Dr Nikhil Nishant, Dr Lalit Sankhe, Dr Chhaya Rajguru \\ Department of Community Medicine, Grant Government Medical College, Mumbai- 400008 \\ Corresponding Author \\ Dr Chhaya Rajguru \\ Email: chhayarajguru@yahoo.com, Phone no: 9820976026
}

\begin{abstract}
Background: Rabies is a 100\% fatal disease affecting all mammals. Dog is the main reservoir and is a major socioeconomic and public health problem. About 59,000 people die annually due to this disease. It affects all and India is no exception, being a classic example of neglected tropical disease. Though effective vaccines are available and prevention is possible by post exposure prophylaxis (PEP), India still lags behind in achieving rabies free society. This is due to poor compliance and lack of awareness in the society. Though fatal the disease is also $100 \%$ preventable.

Objectives: The purpose of this article is to see the compliance rate among the population and study the profile of the patient and animals.

Methods: A follow up longitudinal study was done at Anti-Rabies Vaccination Out Patient Department (ARV OPD) of a Tertiary Care Hospital among patients attending ARV OPD for animal bites fromlst March to $31^{\text {st }}$ October 2015. A detailed patient history was taken and compliance rate was determined based on the ARV register.

Result: All together 1769 patients were followed up. The compliance rate is just above the half-way-mark $(55.2 \%)$ with dog involved in majority of the cases. Maximum were Cat II patients $(76.1 \%)$ with the maximum drop out for the $4^{\text {th }}$ dose.

Conclusion: There is dire need to increase the compliance rate and awareness among the general population. Non compliance is an economic burden on the government treasury and puts society at risk.

Keywords: Anti Rabies Vaccination, Compliance, Animal Bites, Post exposure prophylaxis.
\end{abstract}

\section{Introduction}

Rabies is one of the oldest recognized infectious diseases. ${ }^{(1)}$ It is an acute viral disease which causes fatal encephalomyelitis being $100 \%$ fatal. Rabies affects all mammals, primarily being disease of terrestrial and airborne mammals, including dogs, wolves, foxes, coyotes, jackals, cats, bobcats, lions, mongooses, skunks, badgers, bats, monkeys and humans. The dog has been, and still is, the main reservoir of rabies ${ }^{(1)(2)(3)}$ Canine rabies remains a major socioeconomic and public health problem. The disease claims the lives of an estimated 59,000 people annually, mostly among underserved populations in Africa and Asia. ${ }^{(4)}$

Rabies occurs in more than 150 countries and territories $^{(5)}$. It is a disease entrenched in history, 
dating back to ancient Egypt. With the expectation of some areas in the South pacific, rabies persists as a major Public Health hazard in many countries across the world. ${ }^{(5)}$. It is estimated that around half of the global human population lives in canine rabies-endemic countries and is at risk of exposure. Because cases often go unreported, it is agreed that official records vastly under-estimate the true burden of rabies. ${ }^{(6)}$

Human rabies is endemic in India. ${ }^{(7)}$ In India more than $90 \%$ cases of rabies are due to dog bites. It is one of the most important zoonotic diseases in India and has been recognized in India since the Vedic period $^{(3)}$. As a disease that mostly impacts poor communities, rabies is a classic example of a neglected tropical disease ${ }^{(6)}$

In spite of the long-standing nature of the problem, and despite the presence of effective intervention strategies for rabies control, rabies continues to pose a major public health challenge to program planners. India has not been able to develop sustainable, population-level rabies control strategies like dog immunization and dog population control. ${ }^{(3)}$ Effective vaccines are available to control disease in dogs and large-scale elimination of canine rabies is considered epidemiologically and logistically feasible as well as cost-effective ${ }^{(6)}$ Prevention of rabies is possible by providing the exposed person with the proper post exposure prophylaxis (PEP). PEP in rabies exposed persons includes wound cleaning, post exposure vaccination and administration of rabies immunoglobulin. ${ }^{(2)(7)}$

At the dawn of 21 st century, we have achieved treatments progress in many spheres including science and technology, information technology, agriculture, satellite communication, etc. Unfortunately, we still have the highest number of deaths due to rabies, ironically a disease preventable by modern prophylactic measures. The reasons given for not reporting, completing, or adhering to PEP include poor awareness about the danger of the disease, small size of the injury, reluctance of the dog owner to pay for treatment costs, and not being advised to take PEP. ${ }^{(7)}$
Rabies remains a neglected disease in terms of policy formulations throughout most of the developing countries. ${ }^{(7)}$ Rabies is $100 \%$ fatal, at the same time $100 \%$ preventable if managed appropriately and timely ${ }^{(5)}$. Hence this study was undertaken to know the compliance rate among the patients attending ARV OPD for animal bite cases.

\section{Methods and Materials}

A follow up longitudinal study was done at Anti Rabies Vaccination OPD of a Tertiary Care Hospital among patients attending ARV OPD for animal bites from $1^{\text {st }}$ March 2015 to $31^{\text {st }}$ October 2015 and were advised to receive anti rabies vaccination during the study period. The bite victims were classified and treated as per the WHO guidelines. All subjects who fulfilled the criteria for WHO category II and III exposure and were advised to receive rabies PEP (intradermal) were included in the study.

All Category I patients and patients referred from other facilities for Immunoglobulins (ARS) only were excluded from the study. Also patients who received intramuscular vaccination were excluded. A pretested and predesigned questionnaire was used to elicit the required data pertaining to age, sex, residence (urban/rural), type of dog (pet/stray, observable/non observable, provoked/ unprovoked) site of dog bite marks, diagnosis of bite, status of previously immunized against rabies of cases etc., by interviewing. Records and data of patients were retrieved from the OPD's Animal Bite Registry and Records. Category of bite was determined by attending physician. Follow-ups with regard to the date of a subject's exposure and compliance with the scheduled PEP were made based on the patient's record/chart.

The total number of patients who complied and did not comply with the PEP was determined based on patient's records. The assessment of treatment compliance of PEP regimen was considered on the basis of ARV regimen (intradermal injection of ARV on 0, 3, 7, 28 days). The person, who had received all recommended 
doses of ARV, was considered as completed PEP and who did not receive all recommended doses, was considered as defaulted PEP.

In data analysis the distribution and cumulative number of patients seen and advised rabies PEP for the period of March 2015 to October 2015 were investigated. Frequencies were calculated for the categorical variables. The variables taken into account were ,patient's demographic profiles such as age and sex; exposure characteristics such as category of exposure, site of lesion, number of lesion (single or multiple); status of biting animal; treatment (rabies vaccine and/or immunoglobulin), number of doses administered and compliance.

All Patients coming to OPD for anti rabies vaccination were followed till $31^{\text {st }}$ December 2015 and there compliance was seen. All together 1769 patients where followed up for the study and records analysed. No reference to the participant's identity was made at any stage during data analysis or in the paper. Approval for the study was taken from the Institutional Ethics Committee.

\section{Result and Discussion}

During the study period, a total of 1769 patients fulfilled the inclusion criterion and these were followed up for compliance. Majority of cases $531(30 \%)$ belonged to age group 26-40 years. Paediatric patients formed $15.88 \%$ of the study population. Male patients were $83.2 \%$ compared to $16.8 \%$ female.(Table 1) Dog bites were most common $(87.6 \%)$ followed by cat bites (10.5\%).(Figure 1)

The most common site for bites were lower limbs, left limb $(40.92 \%)$ being slightly more prone than right limb (36\%). (Figure 2)

Most of the bites were by stray animals (86.3\%) and were unprovoked $(85.5 \%)$. Mostly the animals were non observable $(77.4 \%)$ and could not be traced. (Table 2)

As per the WHO criteria most of the patients fell into Category II (76.1\%). Only 6 patients came for pre-exposure prophylaxis. Tetanus toxoid (TT) injection was given to 699 (39.5\%) patients from the centre. Out of 417 Cat III patients $83.93 \%$ received Immunoglobulins and rest had history of previous immunization, hence were not eligible for immunoglobulins. (Table 3)

The compliance rate was $55.2 \%$ i.e only 977 patients out of 1769 completed the full course of ARV. Of the total patients $44.8 \%$ were noncompliant of which almost a third of the patients $31.32 \%$ did not come for the second dose. Majority of the patients $327(41.29 \%)$ missed the last $4^{\text {th }}$ dose. Of the Non-compliant patients only 66 were rescheduled out of which only 28 completed the course.

From the study it is evident that the compliance rate is very low among the population, mainly due to lack of awareness about rabies disease. This is much lower as compared to other similar studies done in the past. In study by Domple et al $\mathbf{7 6 . 5 \%}$ cases completed PEP) ${ }^{(7)}$, Shankaraiah et al (the compliance rate for IMRV was $60 \%$ and for IDRV was $77 \%)^{(8)}$, Krishna et al (compliance was $65 \%)^{(9)}$, Anandaraj et al(compliance $\left.82.6 \%\right)^{(10)}$ and Sumit et al $(62.97 \%$ received completed treatment $)^{(5)}$ had higher compliance rates. It was lowest in study by Seenivasan et al (compliance rate being just $6 \%$ for intramuscular regimen) ${ }^{(11)}$. This is a cause of concern and there is urgent need to increase the compliance rate. Non compliant patients are at risk of having rabies and a threat to themselves and the society.

The patient profile was similar to the other studies with the productive age group forming the bulk of cases with male predominance, (in other studies the male percentage ranges from 67\%-77\%) (7)(9)(10) (11) mainly due to outdoor nature of their activity. Patients of all age groups were found to be bitten by the animals, dog bites again proving to be the main culprit for most of the cases. This is in accordance with the other studies and WHO reports. ${ }^{(4)}$ Stray dogs have become a menace and are mostly untraceable. There is need to control the canine population and its immunization.

The maximum animal bite cases were over lower limb and belonged to Category II, which was 
similar to studies done in the past by Krishna et al and Seenivasan et $\mathrm{al}^{(9)(11)}$ but in contrast study by Shankaraiah et al,Domple et al Sumit et al and Anandaraj et al majority of the patients were of Category III bites. ${ }^{(5)(7)(8)(10)}$

The non compliant population is a burden for the society as well as have risk of contracting rabies due to partial immunization. The vaccine is supplied free of cost by the government and the non compliance leads to wastage of the taxpayers money. In the current study 1663 doses were wasted due to non compliance leading to a monetary loss to the tune of about 1.16 lakh (Rs 350 for vial of 5 doses). This financial loss only includes the direct cost related to the vaccine whereas other costs like syringes. needles, gloves etc are not even taken into account, besides there are indirect costs also involved like manpower, logistics etc. Thus even a single non compliant patient forms a huge economic loss to the health system.

The awareness among general population regarding pre-exposure prophylaxis is very poor as only 6 patients came for it. There is dire need to increase this percentage at least for the high risk groups.

Most of the bites in this study were unprovoked and by stray dogs, in contrast with the findings of other studies where pet dogs were the main cause. $^{(7)(8)(11)}$

The number of patients receiving TT at centre is low compared to study by Krishna et al but a very high percentage of patients received immunoglobulins in contrast to very few people receiving in other studies. ${ }^{(9)(10)}$. This difference in use of immunoglobulin may be due to the fact that ARS is provided free of cost to the patients by the hospital and adequate stock is maintained as to prevent any shortage.

Table 1: Patient Profile of animal bite cases

\begin{tabular}{|l|c|c|}
\hline Variables & Number & $\%$ \\
\hline Age(yrs) & & \\
\hline $0-12$ & 281 & 15.88 \\
\hline $13-25$ & 475 & 26.85 \\
\hline $26-40$ & 531 & 30.01 \\
\hline $41-60$ & 383 & 21.65 \\
\hline$>60$ & 99 & 5.59 \\
\hline Gender & & \\
\hline Male & 1472 & 83.21 \\
\hline Female & 297 & 16.79 \\
\hline
\end{tabular}

Figure 1: Type of Animal bite

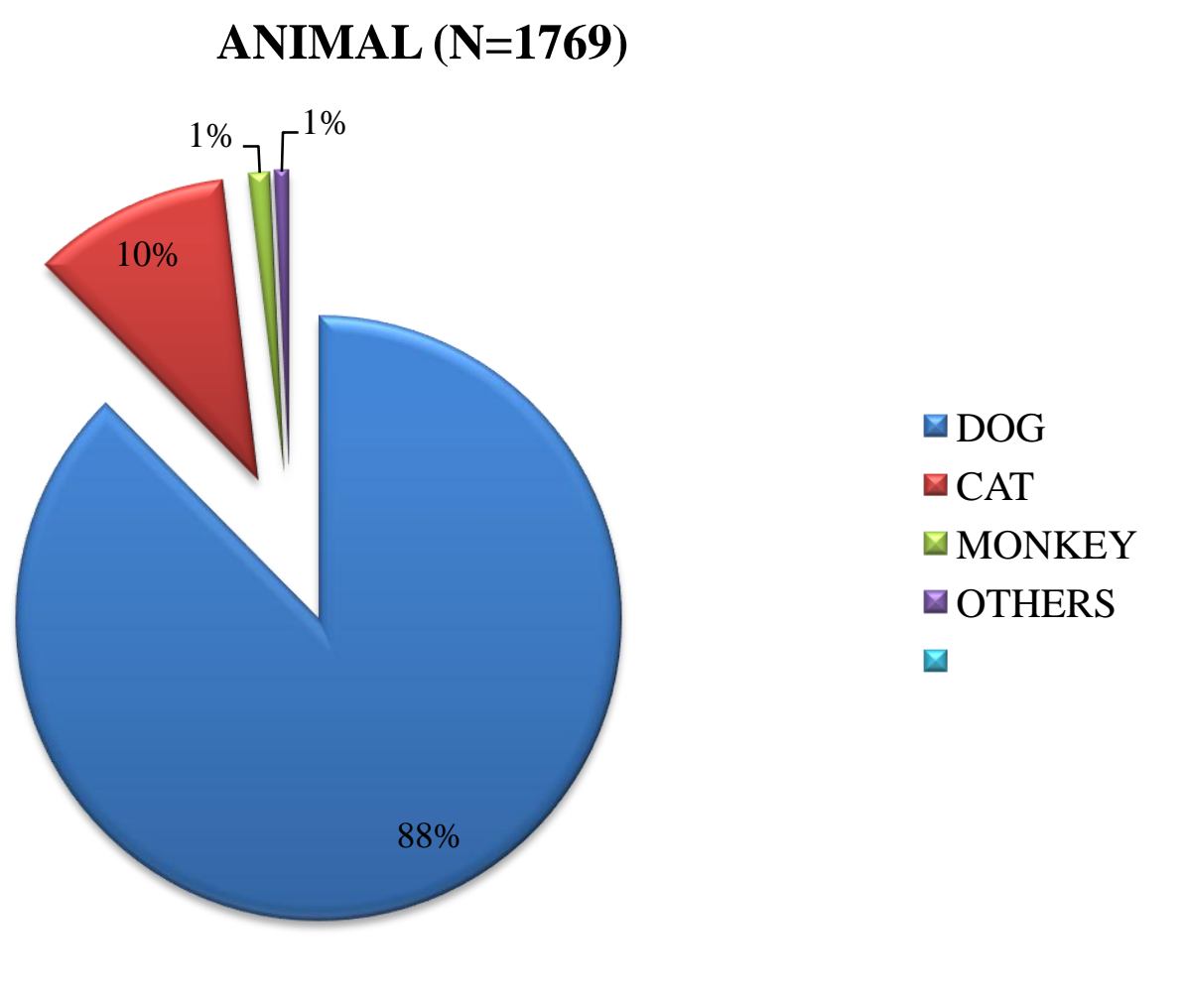


Figure 2: Site of Bite

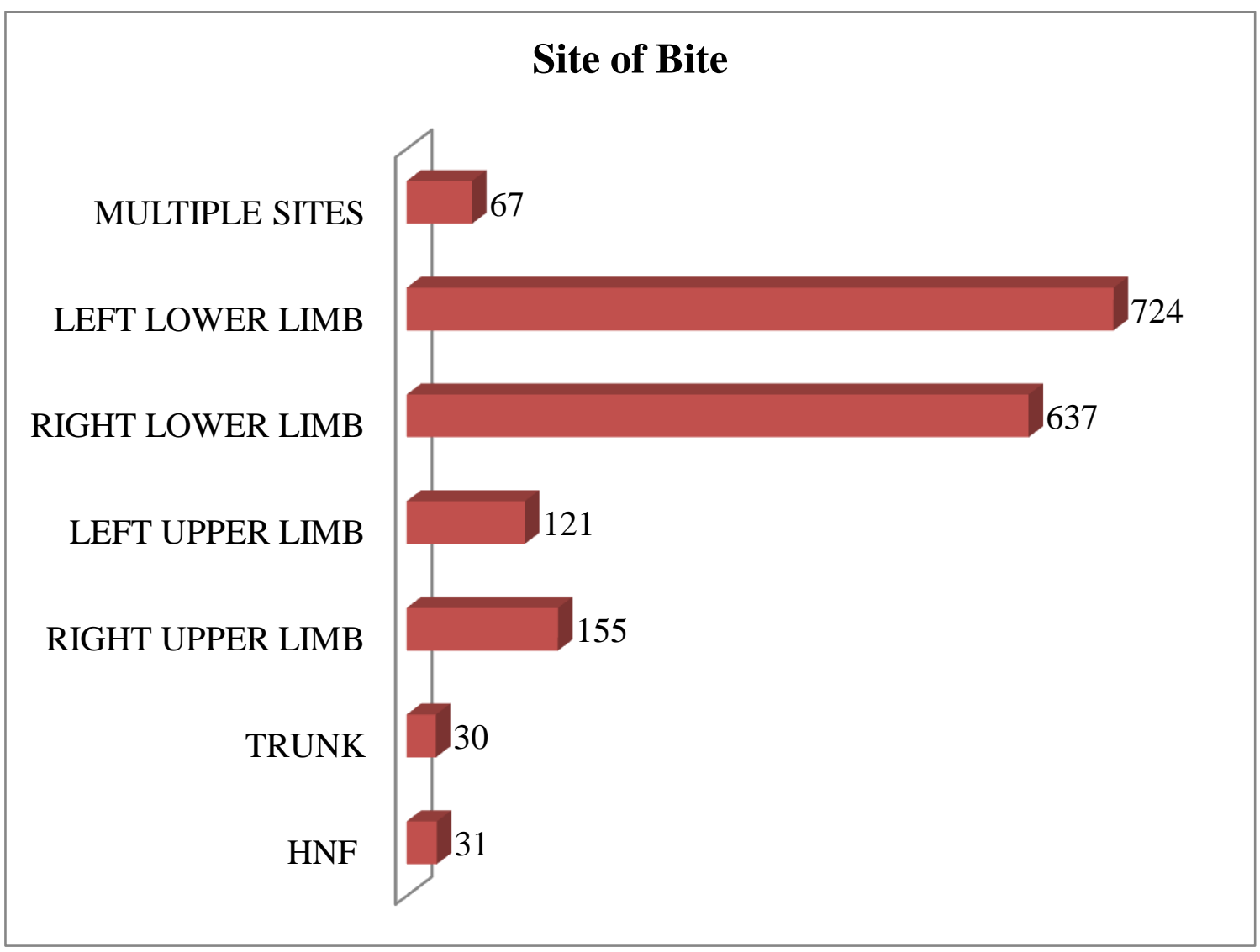

Table 2: Animal Profile

\begin{tabular}{|l|c|c|}
\hline Particular of Animal & Number & $\%$ \\
\hline Pet & & \\
\hline Stray & 237 & 13.4 \\
\hline & & 86.3 \\
\hline Observable & 393 & 22.2 \\
\hline Non observable & 1370 & 77.4 \\
\hline & & \\
\hline Provoked & 252 & 14.2 \\
\hline Unprovoked & 1510 & 85.5 \\
\hline & & \\
\hline Category(severity) & & \\
\hline Cat 2 & 1346 & 76.1 \\
\hline Cat 3 & 417 & 23.6 \\
\hline Pre exposure prophylaxis & 6 & 0.3 \\
\hline & & \\
\hline Animal & & \\
\hline Dog & 1550 & 87.6 \\
\hline Cat & 186 & 10.5 \\
\hline Others & 33 & 1.9 \\
\hline
\end{tabular}

Table 3: Vaccination at ARV OPD

\begin{tabular}{|l|c|c|}
\hline Vaccine & Number & $\%$ \\
\hline Tetanus Toxoid & & \\
\hline Yes & 699 & 39.5 \\
\hline No & 1070 & 60.5 \\
\hline & & \\
\hline Immunoglobulins(n=417) & & \\
\hline Yes & 350 & 83.93 \\
\hline No & 67 & 16.07 \\
\hline & & \\
\hline Compliance & & \\
\hline Compliant & 977 & 55.2 \\
\hline Non compliant & 792 & 44.8 \\
\hline & & \\
\hline Missed Dose(n=792) & & \\
\hline $2^{\text {nd }}$ & 248 & 31.32 \\
\hline $3^{\text {rd }}$ & 217 & 27.39 \\
\hline $4^{\text {th }}$ & 327 & 41.29 \\
\hline
\end{tabular}

\section{Conclusion}

The study provides strong evidence that problem of rabies exposure is widespread in the region with dog being the main culprit. There is ignorance among the patients as to the problems associated with rabies and its severity indicated by the low level of compliance. With the availability of safe and potent vaccines, prophylaxis of rabies 
is possible by immediate and appropriate post exposure treatment.

Proper counselling and follow up system should be developed to increase compliance and to avoid drop out. Training of Health Providers for proper and repeated counselling is necessary. Awareness campaign along with use of media for highlighting the severity of disease and availability of highly effective vaccination should be emphasized. Also awareness needs to be created about pre exposure prophylaxis among the high risk groups.

There is a need to create awareness regarding local wound treatment and adherence to treatment through a strong information education and communication programme among the community.

There is an urgent need to establish a reminder system (may be telephonic reminder messages) to increase the compliance rate and make people aware of the nearest centre for Anti Rabies Vaccination. It is worthy to note that for RabiesPrevention is the Only Cure.

\section{References}

1. Sambo M, Lembo T, Cleaveland S, Ferguson HM, Sikana L, Simon C, et al. Knowledge , Attitudes and Practices ( KAP) about Rabies Prevention and Control: A Community Survey in Tanzania. PloS Negl Trop Dis. 2014;8(12).

2. National Guidelines on Rabies Prophylaxis, National Centre for Disease Control, DGHS, MOHFW Govt of India, 2013.

3. Rangari VD, Patnaik B. Post exposure prophylaxis against rabies with anti-rabies vaccine by intra-dermal route in India: research review MIMER Medical College,

4. World Health Organization. Weekly epidemiological record [Internet]. 2017. Available from: http://www.who.int/wer

5. Aggarwal S, Chaugule R, Haralkar S, Aswar NR, Khandare K, Kumavat AP. A Cross Sectional Study- Intradermal Versus
Intramuscular Anti Rabies Prophylaxis. J Res Med Dent Sci. 2015;3(1):31-4.

6. Kabeta T, Deresa B, Tigre W, Ward MP, Mor SM. Knowledge, Attitudes and Practices of Animal Bite Victims Attending an Anti-rabies Health Center in Jimma Town, Ethiopia. PLoS Negl Trop Dis. 2015;1-14.

7. Domple VK, Doibale MK, Sonkar VK, Aswar NR, Khadilkar HA, Jain SR. Treatment compliance of self-reported dog bite cases attending outpatient department of Tertiary Care Hospital, Maharashtra. Int J Med Public Heal. 2015;5(4):297-300.

8. Shankaraiah RH, Rajashekar RA, Veena V, Hanumanthaiah AND. Compliance to Anti-rabies Vaccination in Post-exposure Prophylaxis. Indian J Public Health. 2015;59(1):58-60.

9. Sahu KK, Manar MK, Singh SK, Singh H. Epidemiological Charecteristics of Patients Attending for Rabies Post- Exposure Prophylaxis at the Infectious Diseases Hospital of Lucknow, India. J Glob Infect Dis. 2015;7(1):30-2.

10. Anandaraj R, Balu PS. Compliance to anti rabies vaccine and animal bite management practices in a rural area of Davangere, Karnataka , India. Int J Community Med Public Heal. 2016;3(1):170-3.

11. Seenivasan P, Caroline PK, Vijayalakshmi M, Saravanakumar P, Jegatheesh S. Anti Rabies Vaccination Coverage Among Patients Attending Outpatient Department In A Tertiary Care Hospital In Tamil Nadu. Int J Med Appl Sci. 2014;3(1):25360. 